\title{
Energy saving design principle analysis of power electronic transformation system
}

\author{
Wan Tao \\ Automotive Engineering Department \\ Henan Vocational Technical Institute \\ Zhengzhou, 450046, China
}

\begin{abstract}
Power electronic transformation system is applied widely in industrial control and the application environment is complex. Big, small and medium-sized system power consumption improves continuously, so it is urgent to reduce the system energy consumption problems. This paper proposes a way to reduce the energy consumption of power electronic transformation system based on genetic algorithm. Work frequency regulation and working voltage measurement technology are used in industrial control system and the voltage and frequency produced by system power consumption are calculated. Genetic algorithm is used to calculate the optimal solution. And then achieve the purpose of reducing energy consumption. Experimental results show that this control algorithm can effectively reduce the power consumption of power electronic transformation system in industrial control and has a good effect.
\end{abstract}

Keywords- power electronic transformation system; Dynamic voltage regulation; Reduce energy consumption

\section{INTRODUCTION}

In recent years, energy consumption problem becomes the difficult and hot problem in power electronic transformation system design, especially in some industrial control system giving priority to COMS circuit. To a certain extent, it restricts power electronic transformation system application and development. In the past industrial control power electronic transformation system design, it mainly involves function, stability, design and production cost etc. [1]. System power consumption is a relatively new design consideration. Now as far as system designers are concerned, apart from considering these properties in the system design process, they are facing a new challenge - to reduce the system real time energy consumption. Reducing the power consumption is based on the comprehensive consideration of extending hand-held devices' battery life, reducing the chip package and cooling costs, improving the system stability and reducing the environmental impact. The importance of the requirements is more and more outstanding with the popularity of handheld devices. The reasons come from the following several factors [2] : first, more and more hand-held devices system uses electric power supply. Reduce power consumption can extend system life; Second, with the rapid development of semiconductor industry, CMOS system integration level and clock frequency increase significantly, which not only leads to the dramatically increased system power consumption, produces the redundant heat release problems, and increases the system packaging costs, but brings huge influence to the stability of the system; Third, energy price rises ceaselessly. Green electrical equipment idea becomes deep in people's heart and people becomes more and more concerned about the environment. This further illustrates the significance of the design of industrial control system to reduce the power consumption. Based on the above factors, reducing the energy consumption of the industrial control system has important practical significance. But most traditional technology reducing power dissipation are based on the hardware circuit design and software development. Reduce unnecessary devices in hardware, and optimize program code structure in software. So the ways reducing the power consumption method can't meet the requirements of industrial control system. More scientific and effective power dissipation reducing technology are needed.

\section{ENERGY SAVING ALGORITHM PRINCIPLE}

There are uneven work load phenomenon in most industrial control power electronic transformation system[3]. Sometimes work system needs full load load, and sometimes load is small. At that time, the master CPU frequency in industrial control system is one of the most important factors in determining system power consumption, in addition the voltage is also a very important reason influencing power consumption.

In today's industrial control environment, most industrial equipments are relevant to CMOS circuit design. The study finds that CMOS circuit has some special properties: the energy consumption of CMOS circuit in the work has positive correlation with the system clock frequency and the system working voltage square. To finish a system CPU's process tasks basically requires fixed time. The energy consumed in the process is proportional to the square of voltage, so we must think of ways to reduce the working voltage of the system, and it will be possible to reduce the energy consumption of the system. But, because the system clock frequency has a linear relationship between working voltage, if the working voltage is reduced, the system clock frequency will also be reduced correspondingly, and the time of completing the task will increase. This may result in the performance decline. The balance scheduling algorithm can change CPU frequency and working voltage when the system is running dynamically, and adjusts the balance between power consumption and performance without affecting the CUP 
peak value performance. The work of reducing energy consumption is completed well.

Working principle diagram is shown in Figure 1 below:

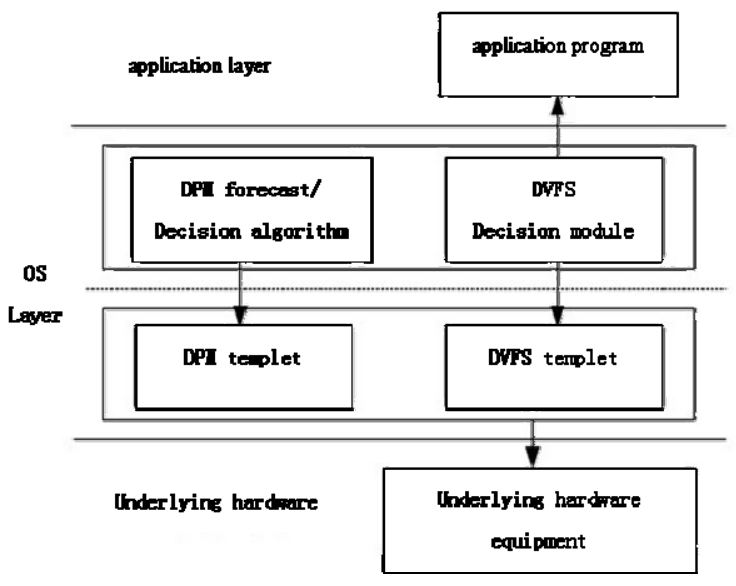

Figure 1. Energy saving principle diagram

\section{VOLTAGE, FREQUENCY OPTIMAL SOLUTION}

System energy saving optimization problem solving is complicated. It is difficult by using general numerical solution way. Based on genetic algorithm, this paper applies adaptive parameter adjustment mechanism in solving power electronic system energy saving optimization problem.

\section{A. Chromosome}

Take system working condition breaking point positions and the corresponding working condition sequence function $\mathrm{W}$ as a chromosome, such as:

$W=\left[\left(S_{1}, G K_{1}\right),\left(S_{2}, G K_{2}\right) \ldots\left(S_{i}, G K_{i}\right) \ldots\left(S_{L}, G K_{L}\right)\right]$

In it, $S_{i}$ is the $i(i=1,2 \ldots \ldots L)$ condition breaking point position, $G K_{i}$ is the $i(i=1,2 \ldots \ldots L)$ the corresponding condition of condition point. $S_{i}$ adopts real number coding mode and the length of the chromosome is $L$.

\section{B. Initialization module}

Generating original population: set the train running condition according to different ramp and typical subinterval's condition setting principle, randomly generate different condition breaking point position, generate $n$ different chromosomes, and form the initial population. The population size is $n$.

\section{Fitness function}

System energy saving optimization is multi-constraints and nonlinear problem. for the solution of convenient, this paper chooses penalty function to transform the original constrained minimum problem into unconstrained maximum value problem.

$$
\begin{aligned}
F_{\text {min }}= & Q+\alpha \times\left(\left|v_{S}\right|+\left|S-S_{\text {set }}\right|+\left|T-T_{\text {set }}\right|+\right. \\
& \text { load } 1+\text { load } 2) \\
F_{\text {max }}= & \frac{1}{F_{\text {min }}}
\end{aligned}
$$

In it,

$$
\begin{gathered}
\text { Ioad } 1= \begin{cases}\phi & \left(v_{i} \geq \mathrm{v}_{\lim , i}\right) \\
0 & \left(v_{i}<v_{\lim , i}\right)\end{cases} \\
\text { load } 2= \begin{cases}\varphi & \left(\frac{d a}{d t} \geq \lambda\right) \\
0 & \left(\frac{d a}{d t}<\lambda\right)\end{cases}
\end{gathered}
$$

$\alpha, \phi, \varphi$ are penalty factors.

\section{Convergence condition}

This paper takes whether achieving maximum iterative number as convergence criteria. If convergence does not appear, continue the following operation, or quit.

\section{E. Genetic operator selection}

Genetic operation includes selection, crossover and mutation.

Select operator: sorting choice. Sorting choice does not use absolute value information of individual fitness function value [10], avoids super individual's absolute influence in population.The specific method of sorting choice is: arrange individuals in group from big to small according to fitness function value, of which the expectation value of each individual selected is $p(j)$.

$$
p(j)=\frac{1}{n}\left(\eta^{+}-\frac{\eta^{+}-\eta^{-}}{n-1}(n-j)\right), j=1,2 \ldots \ldots n
$$

In it, $\eta^{+}+\eta^{-}=2,1 \leq \eta^{+} \leq 2 . n$ is the population size.

Crossover operator: three-point crosses. Choose two chromosomes. Randomly generate a number between $0 \sim 1$. If random number is smaller than the mutation probability $p_{c}$, select the chromosome to cross. While crossing, randomly generate three junctions. While crossing, interchange train condition. In working condition breaking point, select arithmetic crossover operator to cross in order to improve the population diversity, the formula is as follows:

$$
\begin{aligned}
& X_{1 i}^{\prime}=\mu \cdot X_{1 i}+(1-\mu) \cdot X_{2 i} \\
& X_{2 i}^{\prime}=\mu \cdot X_{2 i}+(1-\mu) \cdot X_{1 i}
\end{aligned}
$$

In it, $\mu \in(0,1), X_{1 i}, X_{2 i}$ are the breaking points of the operation condition before cross, $X_{1 i}^{\prime}$ and $X_{2 i}^{\prime}$ are the breaking points of the operation condition after cross. 
Mutation operator: probability variation. Randomly generate between $0 \sim 1$. If random number is less than the mutation probability $p_{m}$, then the chromosome varies. Randomly generate several change points, the working condition of variable point randomly varies.

\section{F. Adaptive mechanism}

In order to improve the convergence and optimization ability of genetic algorithm, this paper introduces the adaptability strategy based on stage evolution:

In the initial stage of evolution, the smaller selection pressure $\eta^{+}$is adopted; larger crossover probability $p_{c}$ and mutation probability $p_{m}$ and population scale $n$, to keep the population diversity.

In the later evolution, group shifts into facing local search and adopt larger selection pressure $\eta^{+}$; lesser crossover probability $p_{c}$ and mutation probability $p_{m}$ and population scale $n$, in order to improve the convergence speed.

This paper chooses adapting strategy based on stage evolution, and divides the whole evolution process into the following three stages:

The first stage: $\left[0, T_{1}\right], T_{1}=\partial T$

$n_{1}=(2-\partial) n, \eta^{+}=1.20, p_{c}=1.0, p_{m}=0.1$

The second stage: $\left(T_{1}, T_{2}\right], T_{2}=(1-\partial) T$

$n_{2}=n, \eta^{+}=1.60, p_{c}=0.75, p_{m}=1 / L$

The third stage: $\left(T_{2}, T\right]$

$n_{3}=\partial n, \eta^{+}=1.80, p_{c}=0.50, p_{m}=0.1 / L$

In it, $\partial=0.382$; $\mathrm{T}$ is the biggest evolution algebra; $\mathrm{n}$ is the biggest population size; $\mathrm{L}$ is individual chromosome length.

\section{G. Algorithm whole block diagram}

Combining with the system energy saving operation algorithm and genetic algorithm, the algorithm whole block diagram is given as shown in Figure 2.

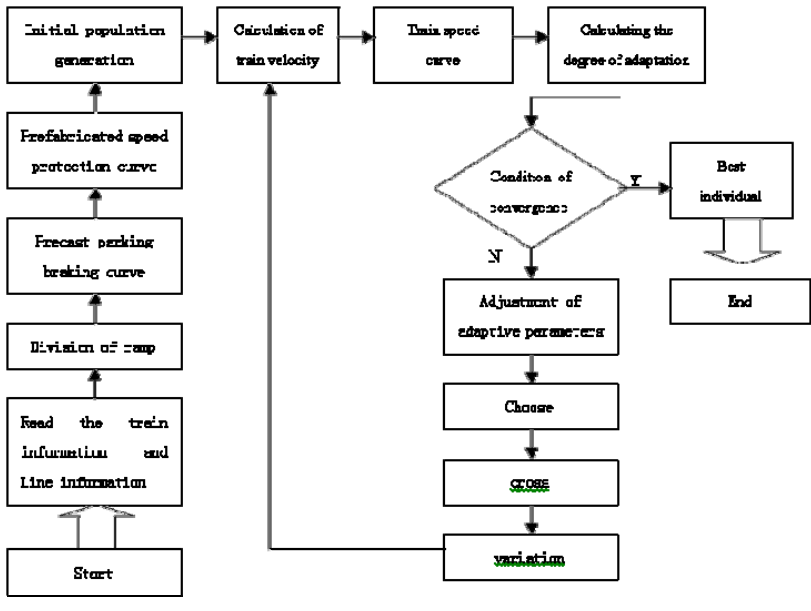

Figure 2. Algorithm whole block diagram

\section{EXPERIMENTAL RESULTS ANALYSIS}

Our balance point energy saving algorithm simulation improves the simulation and evaluation method put forward previously【 5 】. This method takes UI, Real - Time Audio and MPEG Decompression as benchmark programs. We use hardware platform and power electronic controller used commonly in industrial control, ARM7 series processor which can carry out continuous voltage adjustment. The processor has built-in flash memory, I/O control mouth, and can add some external hardware equipments. Emulator inputs a set of consecutive task sequence to test platform. The sequence contains some necessary system parameters. The output is the relation model of processor utilization rate and power consumption. The operating system uses ucos system of better real-time, so we can more clearly observe the changes in energy consumption.

Figure 3 is the ARM7 + ucos power electronic transformation system power consumption got by equilibrium point strategy combining different algorithms get

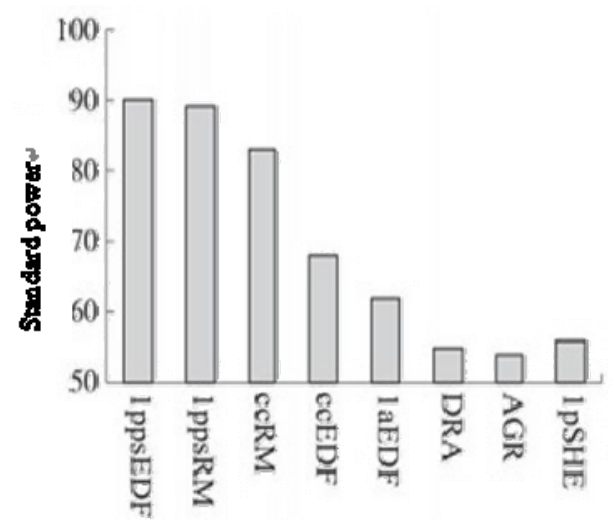

Figure 3. Different algorithms’ power consumption 
To general energy conservation algorithms considering the static power energy saving, when ARM7 utilization is smaller, balance algorithm can obtain the minimum power consumption. With the maximum CPU utilization achieved, general energy saving algorithms have roughly the same power of the strategy of this paper.

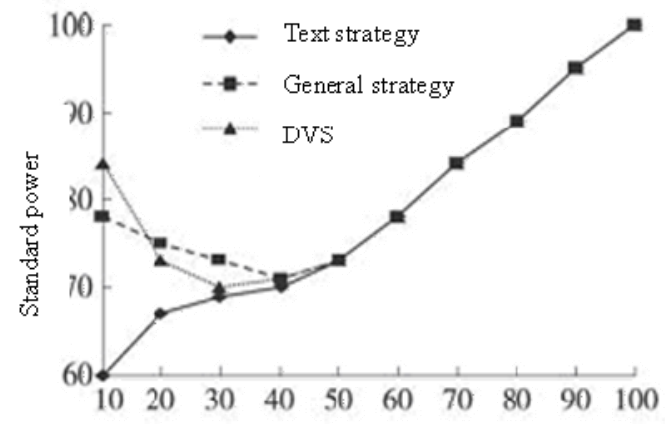

Figure 4. Processor utilization sketch map

The power consumption of Figure 4 is the contrast of this algorithm's balance point algorithm and the standard power consumption without adopting this strategy's power consumption. This strategy can reduce $5 \%$ of the system power consumption compared with conventional energy saving strategy, and as far as the whole system is concerned, the relevant costs are reduced through this strategy so $18 \%$ power saving is obtained than the average energy saving methods.

\section{CONCLUSION}

This paper proposes a way to reduce the energy consumption of power electronic transformation system based on genetic algorithm. Work frequency regulation and working voltage measurement technology are used in industrial control system and the voltage and frequency produced by system power consumption are calculated. Genetic algorithm is used to calculate the optimal solution. And then achieve the purpose of reducing energy consumption. Experimental results show that this control algorithm can effectively reduce the power consumption of power electronic transformation system in industrial control and has a good effect.

\section{REFERENCES}

[1] Mudge T.Power:A first class design constraint for future architecture[C].Proceedings of the 7thInternationalConferenceonHighPerfor-mance Computing,2000.

[2] Jejurikar R, Gup ta R. Energy Aware Task Scheduling with TaskSynchr onizati on for Embedded Real2 Time Systems[J] Computer2Aided Design of I ntegrated Circuits and Systems, IEEE Transac2 tions on, June 2006: 1024 - 1037.

[3] Wang XiaoYing, Zhao Hai, Chen YingGe, etc. Sensor network task double effect energy-efficient scheduling research [J]. Journal of Electronics, 2006, 34 (5) : 798-783

[4] Liu Hao, Bu Aiguo. Low power consumption design strategy of voltage adjustable processor [J]. Journal of Circuits and Systems, 2006, (5) : 44 - 50.

[5] Duan Sheng. A design modeling method of embedded system [J] Computer Simulation. 2007, 1 (25) 